\begin{tabular}{|l|l|l|l|l|l|}
\hline MUNIBE Antropologia-Arkeologia & $n^{\circ} 69$ & $265-281$ & DONOSTIA & 2018 & ISSN 1132-2217 • eISSN 2172-4555 \\
\hline
\end{tabular}

\title{
Relectura arqueológica del pozo-cisterna de la fortaleza de Gijón (Asturias, Norte de España): la civitas Gegionem durante el primer siglo del Reino de Asturias (720-840 AD)
}

\author{
An archaeological reassessment of the well-cistern \\ in the fortress of Gijón (Asturias, Northern Spain): the civitas Gegionem \\ during the first century of the Asturian kingdom (720-840 AD)
}

PALABRAS CLAVES: Registro arqueológico, procesos formativos, Alta Edad Media, centro político, estilo de vida elitista. GAKO-HITZAK: Erregistro arkeologikoa, eraketa-prozesuak, Goi Erdi Aroa, gune politikoa, bizimodu elitista. KEY WORDS: Archaeological record, formative processes, Early Middle Ages, political centre, elite lifestyle.

\section{Alfonso VIGIL-ESCALERA GUIRADO(1)}

\section{RESUMEN}

Una revisión de los datos publicados sobre la estructura hidráulica descubierta en Gjón permite articular una lectura alternativa a la mantenida hasta ahora. Frente a un proceso de abandono plurisecular se plantea la amortización del pozo a consecuencia de un evento traumático durante el último tercio del siglo VIII d.C. El excepcional conjunto de hallazgos que apareció en su interior es congruente con la vinculación del pozo a una autoridad política relevante. Esta circunstancia motiva la discusión sobre el posible papel jugado por la fortaleza de Gijón durante la formación del Reino de Asturias y el paradójico silencio que sobre este centro mantienen las crónicas asturianas entre la retirada de la guarnición musulmana (ca. 720 d.C.) y la primera referencia a la llegada de normandos a la costa asturiana (ca. 844 d.C.)

\section{LABURPENA}

Gijonen aurkitutako egitura hidraulikoaren inguruan argitaratu dituzten datuak berrikusiz gero, orain arte indarrean egon denarekiko ezberdina den irakurketa egin dezakegu. Ehunka urtez abandonatuta egon zela ziotenen ustearen aurka, K.o. VIII. mendeko azken herenean izandako gertakizun traumatiko baten ondorioz zuloa berreskuratu egin zutela planteatu da. Barruan topatutako aparteko aurkikuntza multzoa bat dator zuloa agintaritza politiko esanguratsuarekin lotu izanarekin. Gorabehera horren ondorioz, Gijoneko gotorlekuak Asturiaseko erreinua eratu zenean bete zuen rolaren eta garnizio musulmanak atzera egin (K.o. ca 720) eta Asturiaseko kostaldera normandiarrak iritsi izan zireneko lehen erreferentziaren arteko (K.o. ca 844) garaiaren inguruan Asturiaseko kroniketan gune horrekin lotuta dagoen isiltasun paradoxikoaren eztabaida sortu da.

\section{ABSTRACT}

The review of the published data on the hydraulic structure discovered in Gijon leads to an interpretation alternative to the current one. Instead of a process of abandonment lasting several centuries, we propose that the disuse of the well was the result of a traumatic event happened during the last third of the eighth century AD. The exceptional assemblage of findings recovered inside is consistent with a connection between this well-cistern and a relevant political authority. This fact brings out the discussion about the role possibly played by the fortress of Gijón during the formation of the Kingdom of Asturias and the paradoxical silence maintained about this centre in the Asturian chronicles between the withdrawal of the Muslim garrison (ca. 720 AD) and the first reference to the arrival of Normans to the Asturian coast (ca. 844 AD).

\section{INTRODUCCIÓN}

El pozo-cisterna de Tabacalera es una estructura subterránea de captación y almacenamiento hidráulico localizada durante las intervenciones arqueológicas desarrolladas en el patio de un antiguo convento (reconvertido a mediados del siglo XIX en fábrica de tabaco) del barrio gijonés de Cimadevilla, en el centro de la costa de Asturias. En 2015 se publicaron un artí- culo y un volumen recopilatorio de 350 páginas con una amplia presentación del contexto y los resultados de diversos análisis (Fernández Ochoa et al., 2015e, 2015a). Sobre esos datos se construye el presente trabajo.

La planta de la estructura es casi cuadrada, tiene unos $36 \mathrm{~m}^{2}$ de superficie interior y más de $4 \mathrm{~m}$ de profundidad. Se ha calculado que pudo contener entre 100 y 150 m³ $^{3}$ de agua (Fernández Ochoa et al. 2015d:

(1) aveg@usal.es 
161). Los muros que revisten la fosa muestran por su parte interna dos contrafuertes cada uno (Figura 1). Es probable que dispusiera originalmente de algún tipo de cubierta. La construcción se remonta previsiblemente a época tardorromana.

La estratigrafía documentada en su interior se divide de techo a base en tres bloques: el primero, bastante heterogéneo, se atribuye a la destrucción de la fortaleza ordenada por el rey de Castilla en 1395; el segundo contiene los restos de la demolición de la cresta de los muros del pozo; el tercero y más profundo se divide a su vez en tres capas de limos y arcillas (UUEE 24, 25 y 26, cada una de ellas con casi $50 \mathrm{~cm}$ de potencia) separadas por interfaces muy finas de arena, con algunas piedras. El proceso de formación de esas tres unidades y su cronología centran el debate suscitado en estas páginas.

El presente trabajo se desarrolla en cuatro partes. Primero se evalúa la presencia de una estructura hidráulica como la descubierta en sus coordenadas espacio-temporales. La segunda sección atañe al proceso de formación del contexto arqueológico y resume la lectura publicada acerca de su contenido. En la tercera parte se replantean la cronología y el significado del registro material exhumado. Esta revisión invita a situar la amortización del pozo tras el ecuador del siglo VIII, y no a lo largo de varios siglos, como hasta ahora se ha argumentado. Finalmente se reflexiona sobre las implicaciones históricas de la alternativa propuesta. Se valora el papel jugado por la fortaleza de Gijón en el contexto del surgimiento del Reino de Asturias y se sopesan las posibles causas de su omisión en el relato de las crónicas altomedievales.

\section{EL CARÁCTER ESTRATÉGICO DE LA ES- TRUCTURA HIDRÁULICA}

La existencia de un suministro regular de agua potable es el requisito que hace posible la habitabilidad del cerro de Cimadevilla, un tómbolo rodeado por el mar (Figura 2). El único manantial tradicionalmente explotado (La Fontica o Santa Catalina), todavía activo, suele secarse durante los meses de verano. Tanto es así que el descubrimiento del acuífero subterráneo y la puesta en marcha de un sistema que garantizase la disponibilidad regular de agua dulce constituyen premisas ineludibles para que el enclave haya podido ser habitado de forma estable, adquiriera un carácter defensivo, se dotara de un establecimiento termal (como el atestiguado en Campo Valdés) o incluso que pudiera establecerse la industria de salazones documentada en el istmo (Fernández Ochoa 1994, 1997; Blanco 2003; Sánchez López 2018).

Con los datos disponibles, resulta difícil precisar cuándo se construyó el pozo-cisterna (Fernández Ochoa et al. 2015b: 117). No es descartable que el sistema de captación de agua (ya sea de superficie o del acuífero subterráneo) se modificara a lo largo del tiempo desde el origen del establecimiento gijonés, y que en el cerro hubiera otros pozos no localizados. Sin embargo, una vez fortificado durante el Bajo Imperio, levantada la muralla torreada que cierra el tómbolo por el lado de tierra, y si entendemos que el enclave fue en esencia un polo de poder y gobierno, la probabilidad de que existiera una única estructura principal de aprovisionamiento resulta verosímil. Las razones por las que se habría preferido esa opción remitirían teóricamente a varios supuestos: a) las reservas del

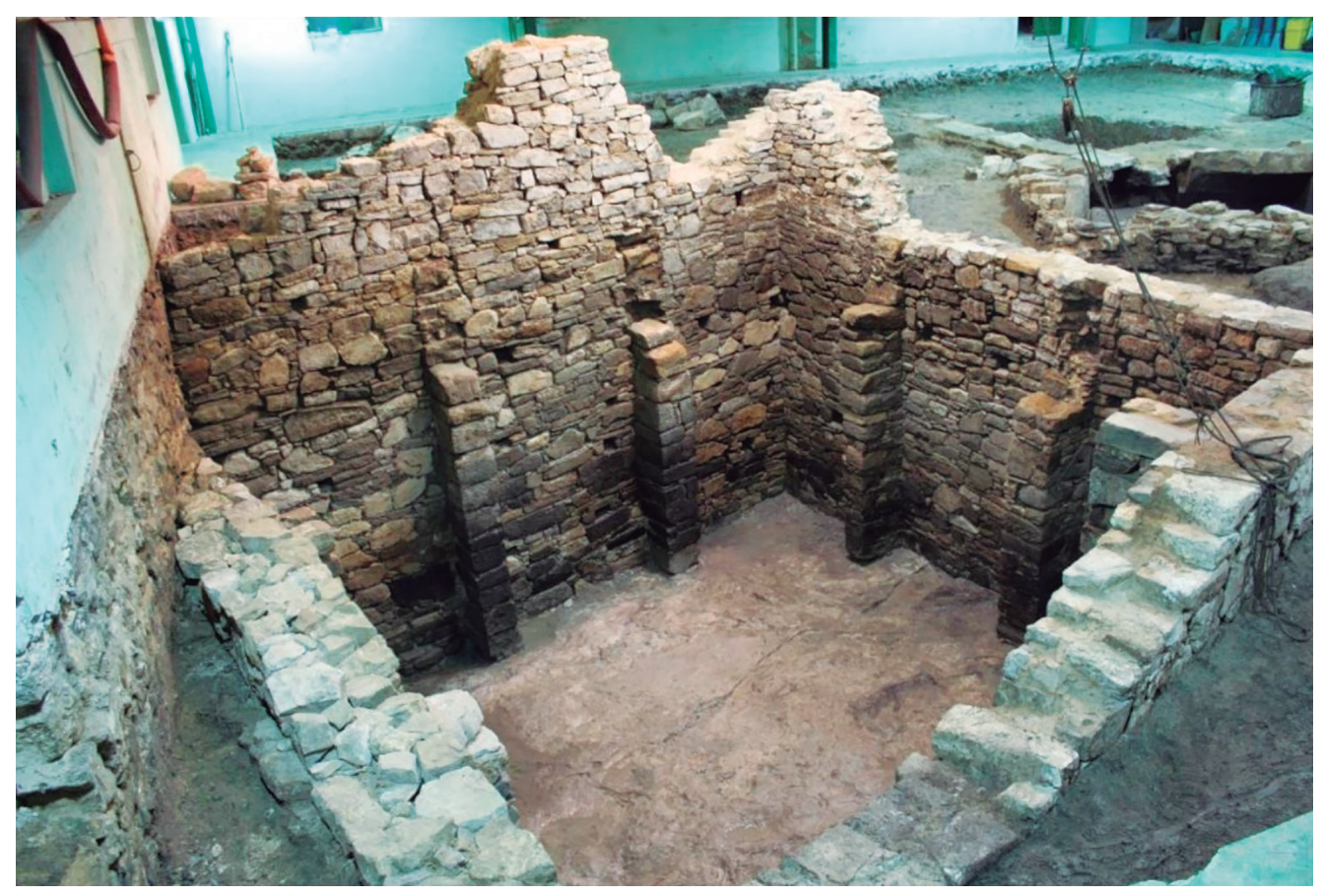

Fig. 1. El pozo-cisterna vacío (FERNÁNDEZ OCHOA et al. 2015: 161, Fig. 2). / The weIl-cistern once empty (from FERNÁNDEZ OCHOA et al. 2015: 161, Fig. 2). 
acuífero eran probablemente limitadas; b) la gestión centralizada de ese recurso garantizaba su calidad y racionalizaba el consumo; c) el control del suministro (especialmente durante el estiaje) suponía el dominio del gobernante sobre los residentes y los eventuales defensores de la fortaleza. Controlar la gestión del abastecimiento de agua era una manifestación no menor del ejercicio del poder y un instrumento para la preservación del orden social.

Tal vez no se haya valorado suficientemente lo que pudo suponer la pérdida de un dispositivo de esa naturaleza. El capítulo del volumen de 2015 dedicado a analizar los recursos hídricos del cerro de Santa Catalina evalúa la calidad microbiológica del agua en distintos puntos (Alonso 2015), pero habría sido útil estimar la capacidad del acuífero y sus límites de recarga, o valorar la posibilidad de que el pozo hallado fuese o no compatible con la existencia de otras estructuras similares. El carácter estratégico de la fortaleza de Gijón o sus meras condiciones de habitabilidad se desvanecerían si sus ocupantes hubieran tenido que salir del recinto para aprovisionarse de agua potable.

En la obra a la que recurrentemente haremos referencia en estas páginas se señala que la amortización del pozo se enmarcaría "en los procesos de transformaciones urbanas detectadas en la ciudad de Gijón a partir del siglo $\mathrm{V}$ y comunes en distintas ciudades hispanas y mediterráneas" (Fernández Ochoa et al. 2015d: 175). La eventualidad de que los datos pudieran sostener una lectura alternativa no llega a formalizarse, siendo ése el hueco que aspira a cubrir el presente trabajo. Se argumentará que el registro arqueológico (puesto a disposición del público con destacable diligencia, lo cual debe ser subrayado) es suficiente para contemplar otra hipótesis: que la inhabilitación pudo tener lugar en un periodo de tiempo muy corto y ser resultado de una acción intencionada ${ }^{1}$.

El envenenamiento del suministro de agua con cadáveres es, de hecho, una práctica bien atestiguada históricamente (Metcalfe 2002: 272; Fischer y Lund 2017: 112). Suele asociarse a acciones de guerra encaminadas a minar las bases del enemigo cuando no existen perspectivas de afianzar el dominio sobre una plaza ${ }^{2}$. Así ocurre por ejemplo en el castillo noruego de Sverresborg, en el que la amortización del pozo tras un asalto fechado en 1197 se produce arrojando a su interior un cadáver ${ }^{3}$. Son ilustrativas también las abundantes referencias de T.E. Lawrence a ese tipo de prácticas

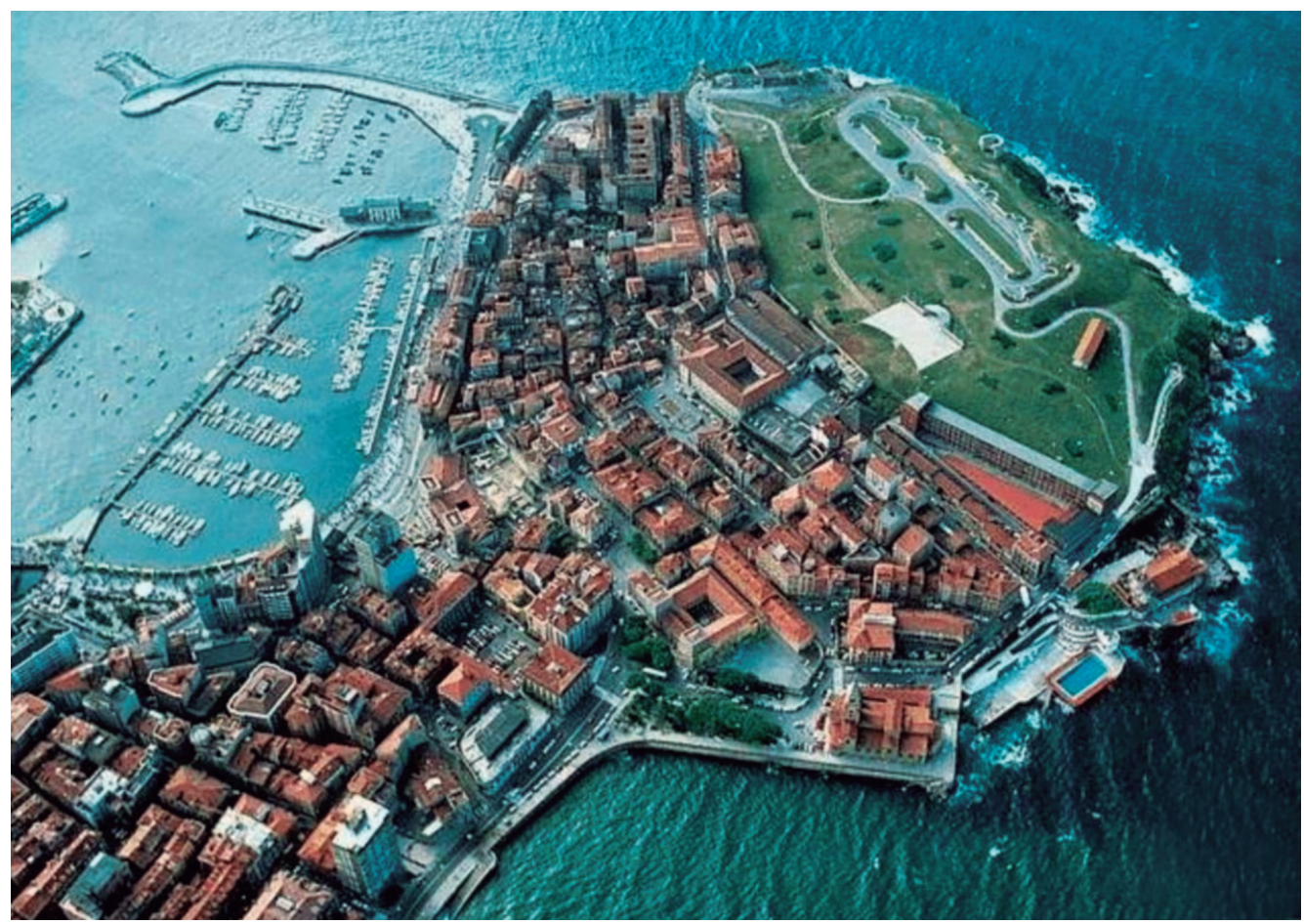

Fig. 2. El tómbolo de Cimadevilla (Cerro de Santa Catalina) en la actualidad. / The tómbolo of Cimadevilla (Santa catalina's hill) at present.

\footnotetext{
El elevado número de cadáveres, los tipos de animales representados, y el contexto y las circustancias que rodean al pozo nos movieron a explorar esta posibilidad.

${ }^{2}$ Envenenar el pozo es también un sintagma empleado en la teoría de la comunicación. Se emplea dicha táctica para silenciar al oponente, violando así su derecho a argumentar sobre un asunto que las dos partes han acordado debatir (Walton 2006).

${ }^{3}$ La excavación arqueológica remite a detalles del relato sobre la toma de la fortaleza narrada en una saga: http://sverresborg.no/arkeologiske-undersokelser, último acceso 06/11/2018.
} 
en Los siete pilares de la sabiduría 4 . Reflexionar sobre las posibles causas de un evento de esa naturaleza puede ayudar a determinar sobre quién recaería la responsabilidad de tal acción, o al menos, a descartar opciones $^{5}$. En cualquier caso, merece la pena considerar que los responsables de una acción de esa naturaleza podrían haber condicionado (tal vez incluso imposibilitado) la habitabilidad de la plaza durante un tiempo.

\section{LA FORMACIÓN DEL DEPÓSITO ARQUEOLÓ- GICO Y SU CONTENIDO}

La trascendencia de analizar y comprender adecuadamente los procesos formativos del registro arqueológico (Lucas 2012; Jiménez Jáimez 2008; Schiffer 1987) se manifiesta de manera bien evidente en este caso. Los aspectos relativos a la formación de la estratificación arqueológica, los procesos postabandono y los tafonómicos comparecen a veces en un segundo plano en el repertorio de contingencias hermenéuticas a nuestra disposición. Como veremos, la lectura de la formación del depósito basal ofrecida en 2015 es sustancialmente unívoca: establece un inicio del proceso en torno a finales del siglo quinto y su desarrollo (la contribución de materiales y sedimento al relleno de la estructura) a lo largo de unos tres siglos, hasta el VIII d.C.

\subsection{La versión publicada: un proceso lento que arranca a finales del siglo quinto}

De acuerdo a los responsables del proyecto, "una vez el pozo dejó de cumplir su función [...] la estructura fue abandonada" (Fernández Ochoa et al. 2015b: 117), siendo así que "el contenido del pozo-depósito de Tabacalera es el resultado del abandono de esta estructura, convertida en vertedero de escombros a partir de finales del siglo V d.C. o inicios del VI d.C. Los materiales [...] fueron depositados en distintos momentos [...] dentro de un arco cronológico que va del siglo $\mathrm{VI}$ al VIII" (Id.: 2015b: 126).

Un examen atento de la documentación permite deducir que los argumentos esgrimidos para defender esta lectura sobre la formación del contexto, su fecha de inicio y el carácter del proceso, proceden de tres frentes. Por un lado se encuentran los materiales contenidos en el pozo susceptibles de datación arqueológica, en especial los fragmentos de vajilla cerámica (Fernández Ochoa et al. 2015c). El estudio de los restantes hallazgos no desentona de las conclusiones obtenidas a partir del análisis ceramológico (Fernández Ochoa et al. 2015e). En segundo lugar se dispone de los análisis sedimentológicos. Finalmente están las dataciones radiocarbónicas de diversos elementos, tanto de objetos arrojados al pozo como de materiales heterogéneos que acabaron formando parte del sedimento (semillas, pequeños restos óseos, materia vegetal). Sus resultados se ofrecen en unas útiles tablas (Fernández Ochoa et al. 2015b: 122-3).

\subsubsection{Cerámica}

El lote de cerámica doméstica recuperado en los tres estratos de relleno más profundos del pozo es realmente escaso para el volumen de los depósitos excavados (no menos de $54 \mathrm{~m}^{3}$ de sedimento). Está compuesto por trece fragmentos, todos menos uno de minúsculo tamaño. Del estrato basal (UE 26) proceden once de ellos (Fernández Ochoa et al. 2015c: 126-8). Un trocito de cuenco de TSHT de la forma 37 tardía, fechado entre mediados del siglo IV y el siglo $\mathrm{V}$ dC y otro de TSAD, tipo D1, de la forma Hayes 91c. El tipo suele fecharse en los decenios centrales del siglo VI (Bonifay 2004: 179). Otro fragmento se asigna a producciones que imitan la terra sigillata gris gálica tardía. Se trata de la base de un cuenco de la forma Rigoir 6 , fechado habitualmente en el siglo $\mathrm{V}$ y la primera mitad del VI (Fernández 2014: 282-4). Se han identificado además un fragmento de cerámica común romana del tipo 'de borde plano inciso', a torno lento, de cronología discutida, otro de pared con espatulado vertical (adscrito al denominado Grupo 5 de la muralla gijonesa) y seis fragmentos asignados al Grupo 10, descrito como cerámica local con vesículas, con factura a torno rápido (Fernández Ochoa et al. 2015c: 128) o a torneta (Requejo 2016: 122), cuyas primeras producciones se situarian entre finales del siglo $\mathrm{V}$ y el siglo $\mathrm{VI}$ dC. (Id.: 125). Tres de ellos pertenecen a una forma cerrada (jarro $u$ olla), uno a un cuenco ${ }^{6}$ y el último a un recipiente con pie trípode, probablemente de producción foránea. Ejemplares análogos aparecen en contextos peninsulares $^{7}$ de los siglos VII a IX d.C., aunque el tipo cuenta con precedentes desde época romano-republicana.

El fragmento de botella de la UE 25 es el de mayor tamaño del lote, aunque le faltan el cuello, las asas y el borde. Presenta decoración incisa de ondas y bandas horizontales a peine en el hombro. La forma es habitual en los repertorios de tradición tardorromana hasta al menos los siglos VII-VIII d.C. De la UE 24, para finalizar, procede el borde de una forma abierta, plato o fuente, con factura a torno rápido y cocción oxidante. Su pasta, según los autores del estudio, recuerda a la de las cerámicas bajomedievales de Gijón (Fernández Ochoa et al. 2015c: 129), aunque "las características morfoló-

\footnotetext{
${ }^{4}$ La obra contiene numerosas referencias a pozos y al envenenamiento de los mismos arrojando cadáveres a su interior (Lawrence 1955: 291). ${ }^{5}$ Una acción de rapiña no suele contemplar tales medidas. Dificultar la recuperación de la comunidad afectada compromete la posibilidad de repetir el saqueo a corto o medio plazo (Scheen 1996: 87-8).

"Sus rasgos "no se han encontrado en contextos anteriores a la mitad del siglo V y perduran sin problemas hasta el siglo VII" (Fernández Ochoa et al. 2015c: 129: 128).
} 
gicas de la pieza y su posición estratigráfica permiten datar la cerámica en tiempos anteriores a la monarquía asturiana en algún momento del siglo VII o quizás inicios del VIII" (Fernández Ochoa et al. 2015c: 129).

Los autores concluyen que "los materiales cerámicos de la UE 26, que es el estrato que se asienta sobre el fondo del pozo-depósito, forman un grupo de cronología homogénea, con una datación que puede fiarse entre finales del siglo $\mathrm{V}$ d.C. y la primera mitad del siglo VI d.C." (Fernández Ochoa et al. 2015c: 129).

\subsubsection{Sedimentología y radiocarbono}

La lectura del proceso formativo de la estratigrafía documentada en el interior de la estructura se sustenta de manera principal sobre el análisis sedimentológico ofrecido en sendos apartados (Jiménez-Sánchez et al. 2015; Sánchez Díaz et al. 2015). Se describe en ambos una secuencia lineal y progresiva de sedimentación cuyo sostén son las dataciones radiocarbónicas obtenidas sobre materiales recuperados en dos columnas que interesan la parte inferior del relleno de la estructura. A partir de los resultados del radiocarbono, se proponen cinco etapas de sedimentación que abarcan desde la construcción del pozo a la serie de rellenos medio-superiores en medio no acuoso. A la vista de los datos se concluye "que el relleno estudiado se produjo entre los siglos VI-VIII" (Jiménez-Sánchez et al. 2015: 188), y que la única muestra de fecha considerablemente anterior correspondería a "material orgánico antiguo que fue transportado al pozo intencionadamente".

Los resultados de las diez dataciones efectuadas sobre diversos materiales procedentes de esas colum- nas geoarqueológicas se ilustran en una tabla que incluye los datos básicos (Id.: 190, Tabla 1, p. 123). De entre ellas merece la pena destacar tres de la UE 26, sobre muestras de materia vegetal, madera y una semilla respectivamente (Tabla I).

\subsubsection{Otros materiales}

El elenco de materiales se completa con diversos objetos de madera: un portaviandas, una fuente y un plato, tres cubos, un travesaño (posiblemente asociado al mecanismo para extraer el agua del interior), parte de una silla y la clavija de un lute. Se han publicado los resultados de los análisis radiocarbónicos efectuados sobre nueve de esas piezas. Las muestras procedentes de la silla y de uno de los recipientes arrojan las fechas calibradas más altas del conjunto ${ }^{8}$ (siglos V-VI para el recipiente troncocónico, y del segundo cuarto del siglo $\mathrm{V}$ al segundo cuarto del siglo VII para la silla). Por el contrario, la datación de una de las fuentes recuperada en la UE 26, apunta a un arco "entre el último tercio del siglo VIII y el inicio del siglo X d.C." (Fernández Ochoa et al. 2015c: 132), mientras que un plato de la UE 25 proporciona una horquilla de probabilidades entre 690 y $880 \mathrm{AD}$

Se recuperaron igualmente restos de al menos cuatro suelas de calzado de cuero, un cesto de fibra vegetal, diversas piezas metálicas (caldero, cuchillo y abrazadera) y material latericio. Especialmente significativos resultan los resultados radiocarbónicos sobre la fibra del cesto, por tratarse de un material de vida más corta que la de la madera y con menos problemas de interpretación de las fechas resultantes (SCHIFFER 1986) (Tabla II).

\begin{tabular}{|c|c|c|c|c|c|}
\hline UE & Código & Material & Ref. Lab. & Fecha convencional & Calibración a 2 sigma \\
\hline 26 & S1-M41 & Vegetal & Beta-333392 & $1330 \pm 30$ & $\begin{array}{l}649-718 \mathrm{dC}(78,5 \%) \\
742-767 \mathrm{dC}(16,9 \%)\end{array}$ \\
\hline 26 & S1-M50 & Madera & Beta-295367 & $1390 \pm 30$ & $602-674 \mathrm{dC}(95,4 \%)$ \\
\hline 26 & TB09.20.83.IIIB & Semilla & Beta-318620 & $1370 \pm 30$ & $608-689 \mathrm{dC}(95,4 \%)$ \\
\hline
\end{tabular}

Tabla 1: Fechas radiocarbónicas de materiales del depósito basal (UE 26). / Radiocarbon dates from materials in the basal deposit (UE 26).

\begin{tabular}{|c|c|c|c|c|c|}
\hline UE & Código & Material & Ref. Lab. & Fecha convencional & Calibración a 2 sigma \\
\hline 26 & TB09.20.83B.74 & Fuente (madera) & Ua-39112 & $1159 \pm 30$ & $\begin{array}{l}775-905 \mathrm{dC}(70,8 \%) \\
915-970 \mathrm{dC}(24,6 \%)\end{array}$ \\
\hline 25 & TB08.20.82.9 & Cesto (fibra) & CNA-561 & $1195 \pm 35$ & $\begin{array}{c}695-700 \mathrm{dC}(0,4 \%) \\
710-745 \mathrm{dC}(6,7 \%) \\
765-900 \mathrm{dC}(83,9 \%) \\
920-950 \mathrm{dC}(4,3 \%)\end{array}$ \\
\hline 25 & TB09-20-82F-18 & Plato (madera) & Ua-39116 & $1233 \pm 32$ & $680-890$ dC (95,4\%) \\
\hline
\end{tabular}

Tabla 2: Resultados del análisis radiocarbónico del primer conjunto de materiales. / Radiocarbon dates from the first set of materials.

\footnotetext{
7 Se citan la necrópolis de La Moranilla, en Écija, y 'horizontes paleoemirales' de Jaén, Cáceres o Guadalajara (Fernández Ochoa et al. 2015c: 129).

8 Todas las calibraciones referidas en el texto remiten a 2 sigma (95,4\% de probabilidad).
} 


\subsection{La versión alternativa: un evento traumá- tico acaecido tras el ecuador del siglo octavo}

Los tres estratos de relleno más profundos del pozo (UUEE 26, 25 y 24) comparten rasgos sedimentológicos y similar potencia, alrededor de medio metro cada uno. El superior apenas presenta inclusiones antrópicas (un fragmento de cerámica fechado entre los siglos VII y VIII d.C.). El más profundo concentra los restos de fauna. Entre todos restituyen un modestísimo lote de fragmentos de cerámica doméstica cuyas fechas extremas irían desde la segunda mitad del siglo IV (la TSHT) hasta los siglos VII-VIII (el recipiente trípode ${ }^{9}$ ). Para los autores del estudio esos materiales indicarían un proceso de relleno lento y progresivo de la estructura.

No obstante, es posible plantear con esos mismos datos un escenario bastante distinto si asumimos que esos estratos profundos de limos (al menos las UUEE 26 y 25, probablemente también la 24) se formaron a consecuencia de un evento de corta duración. Esa circunstancia explicaría mejor el hecho de que a lo largo de tres siglos se hayan incorporado tan escasos fragmentos cerámicos al contexto: serían simple ruido de fondo, material residual. Su significado equivaldría al del hallazgo del fragmento de TSH altoimperial en uno de los rellenos superiores del pozo (UE 18, formada en época bajomedieval).

Determinar el momento de formación de un depósito arqueológico requiere apoyarse en la fecha más moderna proporcionada por los materiales incluidos en el mismo. Este es un aspecto crucial de todo el argumento que viene a continuación y concierne igualmente a las fechas obtenidas del resto de materiales sometidos a análisis radiocarbónicos. Las leyes estratigráficas estipulan que el material más moderno presente en un determinado contexto es el que ofrece el terminus post quem más próximo al momento de su formación. En el caso de los estratos más profundos de relleno del pozo, el material más moderno puede reconocerse en las dataciones radiocarbónicas ofrecidas por ciertos materiales y en algunos de los fragmentos cerámicos recuperados.

\subsubsection{La cerámica}

Si para determinar la fecha del contexto (y por consiguiente el momento de inutilización del pozo) hubiéramos de atenernos en exclusiva al registro cerámico, lo cierto es que el análisis del heterogéneo lote de fragmentos sugeriría que el primer relleno del pozo se formó entre los siglos VII u VIII d.C. La pieza más relevante sería el recipiente trípode al que antes se ha aludido, procedente del estrato basal. La escasez de piezas similares en contextos bien fechados impide precisar su cronología todo lo que sería deseable, aunque el inconveniente atañe a las producciones cerámicas de este momento en todas las regiones peninsulares (Alba, Gutiérrez Lloret, 2008; Vigil-Escalera, Quirós, 2016). En todo caso, parecería prudente asignarle una fecha a caballo entre los siglos VII y VIII d.C. Sería el material más moderno del conjunto, y por tanto, el que establecería de manera taxativa el umbral temporal a partir del cual se formó el primer relleno del pozo.

\subsubsection{Las dataciones radiocarbónicas}

El análisis de las dos docenas de determinaciones radiocarbónicas publicadas en el trabajo de 2015 merece algunas observaciones (Figura 3 ). Trece han sido realizadas sobre distintos materiales procedentes de la excavación, y otras diez de muestras obtenidas en los sondeos geo-arqueológicos. Quince proceden de la UE 26, ocho de la UE 25 y tres de la UE 24. La madera fue el material elegido para un total de diez análisis, el cuero lo ha sido en tres ocasiones, y materia vegetal sin especificar fue el material muestreado en otras cinco. La selección del primer lote de trece muestras apura por tanto las posibilidades de fechar distintos objetos, aunque a posteriori, tal estrategia podría haber escatimado la opción de resolver las incertidumbres sobre el proceso formativo del contexto. No se han analizado los restos óseos de los animales que aparecieron completos en el pozo. Dado que remitirían a la fecha de su muerte, esos huesos habrían sido un material apto para determinar la posible contemporaneidad del conjunto formado por las carcasas (22 perros y un meloncillo) y clarificar la cronología y circunstancias del evento.

Los resultados de los análisis radiocarbónicos ofrecen dificultades para desarrollar una lectura que no sea la de la historia particular de cada objeto. Elementos como los cubos, la silla o la sofisticada vajilla lígnea pueden haber estado en uso durante un periodo de tiempo prolongado, y a ello hay que sumar que el resultado del análisis no remite a la fabricación, el uso o la amortización de las piezas, sino a la tala del árbol del que procede esa madera, con todas las dificultades que ello comporta (Schiffer 1986).

Por cuanto se refiere a los análisis efectuados sobre muestras de vida corta, hay dos a destacar: una semilla procedente de las columnas geo-arqueológicas y la fibra vegetal de la espuerta. La semilla analizada (TB0920-83-IIIB, Tabla I) procede del sedimento y, por tanto, podría haber formado parte de un contexto estratigráfico distinto a la UE 26 antes de terminar en el pozo, cuando éste se encontraba aún en uso. La fibra del cesto o espuerta es un material idóneo para la datación por radiocarbono, susceptible de proporcionar una fecha cercana en el tiempo a la de la formación del estrato.

\footnotetext{
${ }^{9}$ Estos jarritos (o botellas), a veces pintados con trazos rojos, podrían ser en realidad más característicos del siglo VIII que del VII (Amorós 2011), y muestran vínculos con piezas de época emiral temprana (Serrano et al. 2016).
} 
El cotejo de los resultados ordenados de acuerdo a su posición estratigráfica (Figura 3) no permite apreciar en realidad una secuencia coherente de deposición para los tres estratos. Excluido el resultado de la muestra de sedimento orgánico que ofrece fechas alto y medio-imperiales (siglos II-III d.C.), encontramos fechas altas (antiguas) en las tres unidades, pero también fechas bajas (modernas) en las UUEE 25 y 26 . La situación es análoga a la observada antes con la cerámica.

Una lectura meditada del conjunto de resultados aconsejaría atender en primer lugar a las fechas más bajas obtenidas y, dentro de las horquillas de probabilidad estadística que ofrecen, seleccionar el tramo en el que se produce un mayor solapamiento con las inmediatamente anteriores. Los materiales con las fechas más modernas del lote (Tabla II) son la fuente de madera de roble y la fibra vegetal del cesto. El resultado de la fuente ofrece un arco bastante amplio con un 95,4\% de probabilidades de situarse entre 775 y 970 AD. La del cesto se movería con idéntico porcentaje de probabilidad entre 695 y 950 AD (con un 83,9\% entre 765 y 900 AD). Los materiales con fechas inmediatamente previas a éstas (Tabla III) serían el portaviandas, uno de los cubos y dos de las muestras de materia vegetal de las columnas sedimentológicas (S1M02 y S3M08). Los extremos de las horquillas de fechas calibradas se sitúan en 650 y 780 AD.
El resultado de aquilatar en la medida de lo posible ambos bloques de resultados conlleva por tanto asumir que la estructura hidráulica habría estado todavía en funcionamiento durante la primera mitad del siglo octavo y que el evento desencadenante de su amortización tuvo una alta probabilidad de acaecer durante el último tercio de esa centuria.

\subsubsection{Los análisis faunísticos}

Los estudios arqueozoológicos ofrecen pistas adicionales para determinar cómo se pudo formar el depósito y el contexto social del que proceden los restos.

El conjunto de 22 perros ocupa un capítulo específico de la obra (Llorente et al. 2015a). Por un lado se señala que "la incorporación de perros al depósito lo fue de carcasas enteras" (Morales et al. 2015: 240), circunstancia que sería "más difícil de postular en el caso de las restantes especies domésticas" (Llorente et al. 2015a: 227). Por otro, se advierte la heterogeneidad del conjunto de canes documentado en la UE 26, donde se reconoce la presencia de razas de talla media-grande (protomastines destinados al cuidado de rebaños), de ejemplares similares a los galgos o podencos (para la caza) e incluso de ejemplares de pequeño tamaño (bien para la caza o mantenidos como animales de compañía) (Llorente et al. 2015a: 235). La anomalía de

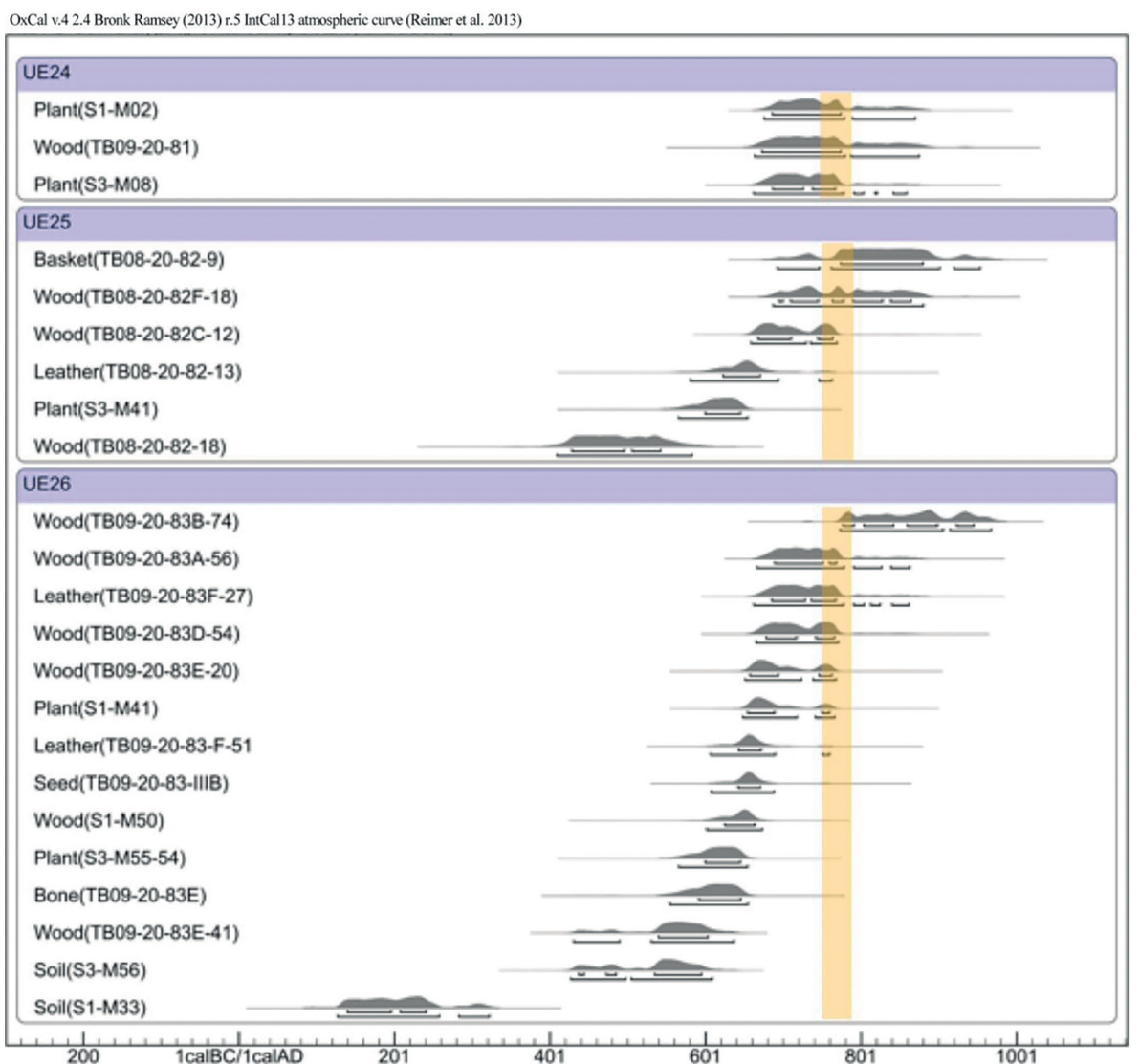

Fig. 3. Tabulación de las 23 dataciones publicadas. Se destaca el intervalo de mayor convergencia (elaborada por M.I. García-Collado). / Tabulation of the 23 published dates. The highest probability interval is highlighted (made-up by M.I. García-Collado) 


\begin{tabular}{|c|c|c|c|c|c|}
\hline UE & Código & Material & Ref. Lab. & Fecha convencional & Calibración a 2 sigma \\
\hline 25 & TB09-20-82c-12 & Portav. (madera) & Ua-39111 & $1301 \pm 30$ & $\begin{array}{l}660-730 \mathrm{dC}(64,6 \%) \\
735-770 \mathrm{dC}(30,8 \%)\end{array}$ \\
\hline 26 & TB09-20-83E-20 & Cubo (madera) & Ua-39115 & $1320 \pm 31$ & $650-780 \mathrm{dC}(95,4 \%)$ \\
\hline 24 & S1M02 & Mat. vegetal & Beta-333391 & $1310 \pm 30$ & $\begin{array}{c}655-730 \mathrm{cal} A D(68 \%) \\
740-770 \mathrm{cal} A D(27,4 \%)\end{array}$ \\
\hline 24 & S3M08 & Mat. vegetal & Beta-333393 & $1320 \pm 30$ & $\begin{array}{c}650-725 \mathrm{cal} \text { AD (73\%) } \\
740-770 \mathrm{cal} \text { AD (22,4\%) }\end{array}$ \\
\hline
\end{tabular}

Tabla 3: Resultados del análisis radiocarbónico del segundo conjunto de materiales. / Radiocarbon dates from the second set of materials.

tal circunstancia induce a los especialistas a sugerir la contemporaneidad de su fallecimiento y el contexto social elitista o aristocrático en el que sería apropiado hallar una mezcla así ${ }^{10}$ (Morales et al. 2015: 244).

A lo anterior se suma la evidencia proporcionada por el meloncillo o mangosta egipcia, incorporado completo a la UE 26 junto con la jauría. Identificado como ichneumon en textos latinos y de época moderna, en Egipto se adaptaban las crías a la cautividad en ambiente doméstico por su habilidad en la caza de roedores y como mascota (Llorente et al. 2015b: 249). De acuerdo al análisis zooarqueológico, se trataría a todas luces de un animal exótico importado del Norte de África ${ }^{11}$.

El estudio realizado sobre los restos de peces señala la presencia de una maragota y cuatro besugos en el depósito basal (UE 26). Dado que estos últimos presentan la misma talla, aparecen con escamas y se documentan restos de sus frágiles huesos craneales, se sugiere que fueron pescados y posteriormente desechados sin haber sido consumidos, probablemente porque se malograron (Roselló, Morales 2015: 254). Es evidente que no se trata de un comportamiento habitual ${ }^{12}$ ni lo que podría esperarse del material procedente de desechos domésticos a lo largo de un periodo de tiempo prolongado durante siglos. La talla de todos los ejemplares sugiere su captura durante el invierno (de noviembre a marzo), periodo en el que previsiblemente se produjo su incorporación al depósito arqueológico. El dato coincide con los resultados del análisis de los restos de aves (Llorente et al. 2015a: 234).

Por lo que respecta al estudio realizado sobre los moluscos, se constata la presencia de lapas (dominantes), además de mejillón, ostra, tritón y gasterópodos terrestres (cuya presencia sería anecdótica). La malacofauna apunta a un marisqueo desarrollado como actividad marginal, si bien llama la atención la presencia de tres ejemplares de tritón, especie apreciada e infrecuente en la actualidad. A diferencia de lo indicado en los apartados correspondientes a los perros y el meloncillo, "interpretar estos hallazgos en clave de consumo de élites resultaría aventurado" (Llorente, Morales 2015: 263).

\subsubsection{Los análisis sedimentológicos}

Por lo que respecta a la sedimentología, los responsables del segundo de los capítulos dedicados al asunto señalan que "la cronología de los estratos de abandono es, de suelo a techo, de más antiguo a más moderno. Se inician las dataciones entre el siglo $\mathrm{V}$ y mediados del VI, hasta llegar al menos al siglo VIII" (Sánchez Díaz et al. 2015: 194). Esa afirmación, sin embargo, no termina de ajustarse al complejo panorama que ofrecen los datos cuando se coteja la información disponible. Unas páginas antes, por ejemplo, se lee que dos de las muestras procedentes de la parte más profunda de los sondeos practicados ofrecen fechas que solapan con las de tramos superiores. Son concretamente la S1M50 (calibrada a 2 sigma entre 599-668 AD) y la S3M55-54 (561-651 AD). En el texto se sugiere "una alta velocidad en la sedimentación" (Jiménez-Sánchez et al. 2015: 189) como explicación a la no estricta concordancia de los resultados de las dataciones con el modelo de deposición progresivo en medio acuoso. El segundo trabajo (Sánchez Díaz et al. 2015: 194-5) contempla la opción de que la estructura se abandonara porque no cumplía ya su función a consecuencia de la apertura de otro pozo de captación en sus inmediaciones, o debido a un incremento de las precipitaciones durante el Periodo Frío Altomedieval (465-890 d.C.), que arrastraría mayor cantidad de sedimentos (Id.: 2015: 198).

Careciendo de una formación especializada en el campo de la sedimentología, no sería prudente por nuestra parte discutir los resultados de ambos estudios. Sin embargo, dado que sus conclusiones derivan sobre todo del cotejo de dataciones radiocarbónicas en columnas de sedimentación que se presumen progresi-

\footnotetext{
10 “La presencia de morfotipos extremos se viene relacionando sistemáticamente con las clases sociales altas (...) lo que podría sugerir que el núcleo de Tabacalera atestigua la presencia de un entorno 'elitista'” (Morales et al. 2015: 244).

${ }^{11}$ Carecería de trascendencia que el contexto se fechase antes o después de la conquista islámica de la Península iniciada el año 711 (Llorente et al. 2015a: 246), al margen de que tras esa data la especie haya podido ser importada con mayor asiduidad. Las relaciones comerciales de la costa cantabro-atlántica con el Mediterráneo oriental antes del siglo VIII d.C. están bien atestiguadas arqueológicamente (FERNÁNDEZ 2014). 12 "Resulta de todo punto chocante si hablamos de restos de comida" (Roselló, Morales 2015: 253).
} 
vas e inalteradas, el análisis podría apriorísticamente estar sometido a cierta circularidad argumental.

Dentro del esquema interpretativo asumido, los cadáveres de los 22 perros habrían ido incorporándose durante trescientos años a los depósitos que colmatan el pozo, lo que entra en contradicción con las observaciones de los arqueozoólogos. Por otra parte, las condiciones de sedimentación en el interior de la estructura habrían sido bien distintas en el supuesto de que esos animales hubieran sido arrojados al mismo tiempo. El análisis sedimentológico no contempla esa eventualidad, de forma que difícilmente podría objetivar, por ejemplo, la distorsión estratigráfica que resultaría del drástico cambio de volumen ocupado por el depósito inferior durante las distintas fases que atravesaría la descomposición de los cadáveres acumulados en el fondo (véase p.ej., KACKI \& alii 2004) (Figura 4).

\section{VALORACIÓN DEL CONTEXTO}

\subsection{Un conjunto significativo}

Los hallazgos del interior del pozo gijonés han sido objeto de minuciosos análisis. Su contraparte, el significado del contexto a partir de la visión integral de las asociaciones que en él se registran, es todavía un aspecto de rasgos difuminados. La relectura del contexto arqueológico defendida en estas páginas trasciende el valor individual de los distintos materiales (cerámica, recipientes, fauna) con el propósito apurar en clave holística el significado integral del lote. Desde este enfoque cobra fuerza la hipótesis de un contexto social elitista o de carácter principesco de fecha altomedieval que hasta ahora solo había sido sugerida en los análisis arquozoológicos.

El hallazgo en el pozo del meloncillo, una mascota exótica originaria del Mediterráneo sudoriental, podría responder a distintas causas. Tal vez se deba a la circulación de regalos habitual entre personajes de relevancia social o política (intercambios dipomáticos). Así lo sugieren los arqueozoólogos, señalando que la especie no se reproduce en cautividad, por lo que estos ejemplares debían ser capturados y habituados a la convivencia con humanos siendo cachorros. El nicho ecológico de ese animal en el Nordeste de África (Egipto) daría una idea acerca del notable alcance geográfico de esas relaciones. Algunas alternativas a su llegada directa a Gijón desde las más alejadas costas del Mediterráneo podrían ser las siguientes: que el dueño del ejemplar procediera de algún punto de la ruta entre el nicho original de la especie y su destino, o que el animal le hubiera sido arrebatado a su propietario original a consecuencia de una acción militar o de saqueo. En cualquier caso, la longevidad de estos animales no suele superar los 8-10 años, de forma que su carcasa en el pozo obliga a remitir su captura y traslado a Occidente en fechas relativamente cercanas en el tiempo a la oclusión del mismo.

Algunas de las piezas de vajilla de madera recuperadas (como el portaviandas) podrían relacionarse sin dificultad con la celebración de banquetes, y poco tienen que ver con el mero carácter utilitario de los platos. Los cubos, elaborados a partir de distintas clases de madera, aparecen con cierta frecuencia en necrópolis altomedievales, aunque en este caso podrían vincularse tal vez al simple trasiego de líquido. a

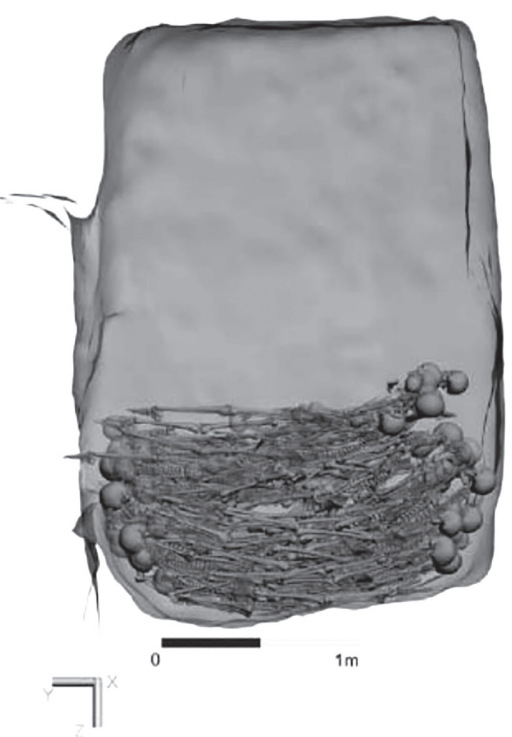

b

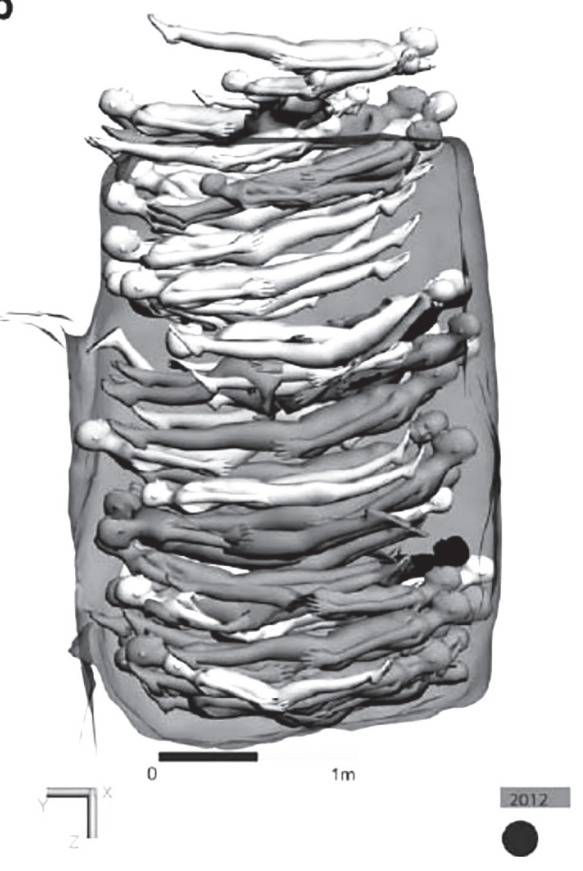

Fig. 4. Cambio de volumen producido por la descomposixión de cadáveres dentro de una estructura-contenedor (de KACKI et al. 2014: Fig. 3). / Volume change produced by decomposing corpses inside a container-structure (from KACKI et al. 2014: Fig. 3). 
El hallazgo de una silla con respaldo y apoyabrazos en un contexto de esta época tiene un sigificado muy distinto del que en la actualidad recibe esa pieza de mobiliario, encontrándose bastante más cerca de lo que consideraríamos una cátedra o un trono. Se trata de un elemento común en las representaciones de reyes, altos dignatarios, obispos o aristócratas (Wanscher 1980). Sentados reciben los gobernantes a sus deudos e invitados, se imparte justicia y se dictan sentencias. Por modestos que puedan parecer sus restos (téngase en cuenta que ésta presenta tanto el respaldo como las patas y el apoyabrazos labrados a torno), la silla es un elemento asociado al ejercicio del poder. En contextos del siglo $X$ de la ciudad sueca de Birka, por ejemplo, algunas sepulturas principescas presentan al fallecido sentado en una silla de madera (Price 2012: 83). Una silla apareció junto a otros numerosos objetos de prestigio en la sepultura de un niño perteneciente a la dinastía real franca enterrada bajo el coro de la catedral de CoIonia (Willemsen 2015: 20, Fig. 10).

No menos sorprendente ha sido la identificación de una pieza de un instrumento musical de cuerda pulsada: la clavija de un laúd o lute. El hallazgo de esta clase de elementos es excepcional en registros arqueológicos. El descubrimiento resulta bien elocuente del tipo de actividades extraordinarias que tenían lugar en las instalaciones de las que formaba parte el pozo.

En el contexto analizado confluyen pues la riqueza, los símbolos de prestigio y de interacción con lugares lejanos o las formas de comportamiento y de consumo conspicuo (Loveluck 2009, 2011; Quirós 2013) con una localización tan excepcional como la que brinda la fortaleza de Cimadevilla (Figura 5). La conjunción de todos esos elementos invita a atribuir al sitio del que forma parte el pozo y al núcleo social allí residente un carácter netamente aristocrático.

Si de nuevo regresamos a la lectura de la formación gradual del depósito, parece francamente complicado de justificar que sólo se hayan arrojado al pozo los cadáveres de esos 22 perros a lo largo de tres siglos ${ }^{13}$. La relectura que aquí se propone sobre la formación del contexto (que el relleno de la estructura arranca de un acontecimiento de carácter puntual) hace que los datos de la fauna hallen acomodo con el resto. El conjunto de hallazgos conduce ahora a un escenario viable según el cual la amortización del pozo supuso la destrucción intencionada de un dispositivo crucial para la fortaleza de Gijón y la de una parte de los bienes del linaje que regentaba ese enclave a mediados del siglo VIII. EI meloncillo y la jauría eran propiedad de alguien con el poder y los recursos suficientes para mantener contactos diplomáticos o comerciales con territorios lejanos, alguien que utilizaba un trono (la silla) para presidir audiencias o tribunales, que organizaba o promovía cacerías y banquetes (como atestiguaría el sofisticado portaviandas), y que además gozaba del entretenimiento proporcionado por músicos o se servía de ellos para agasajar a sus invitados (el laúd).

\subsection{Las ausencias: interrogantes}

El análisis ha estado monopolizado hasta ahora por los materiales conservados y recuperados en el depósito. Cabe preguntarse no obstante por qué razon no han aparecido otros que podrían haberlo hecho en contextos semejantes de esa época. Falta por ejemplo cualquier evidencia acerca de la vajilla de vidrio, es muy escasa la metálica y simplemente anecdótica

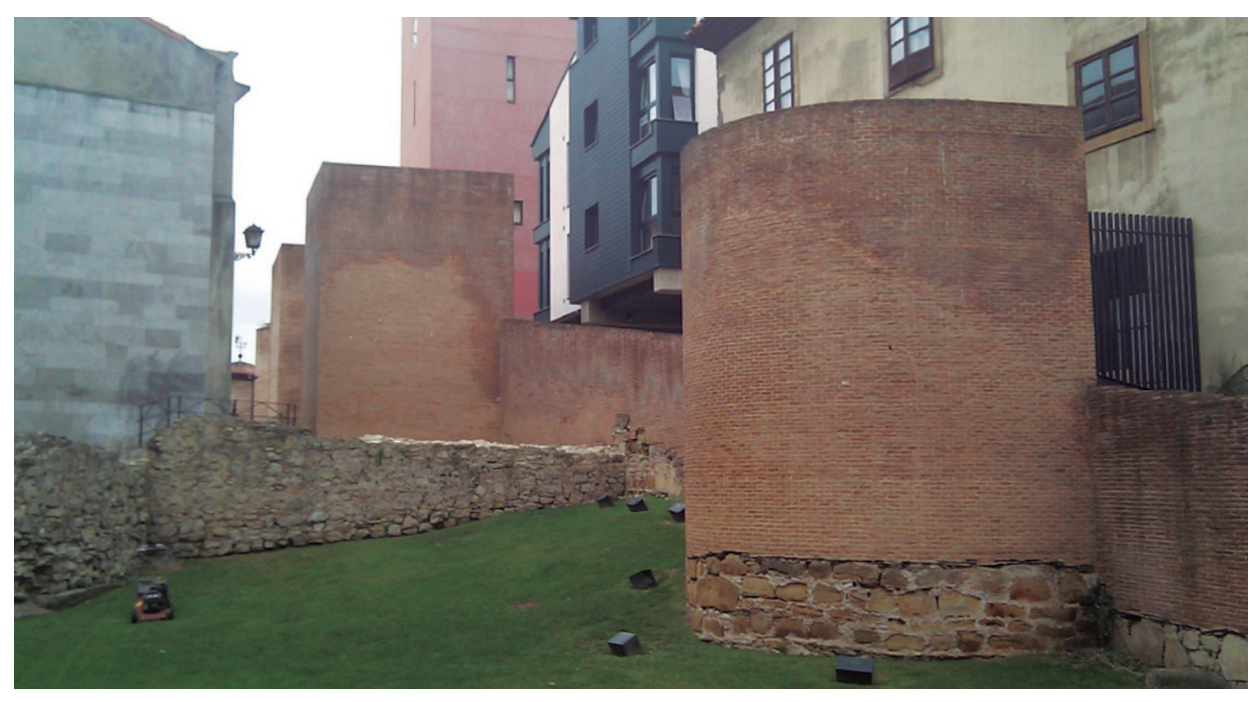

Fig. 5. Restos originales y recrecidos de la fortificación gijonesa. / Original and recreated remains of the walled circuit of Gijón.

\footnotetext{
${ }^{13}$ Hay que hacer constar que también se identificaron cuatro gatos (Llorente et al. 2015a: 232). Otros animales completos, en su mayoría pequeñas aves silvestres o micromamíferos, pudieron fallecer al quedar atrapados en el interior de la estructura tras su inicial amortización.
} 
la presencia de cerámica de mesa o cocina que pudo utilizarse en momentos previos a la amortización de la estructura, pero también durante el posterior proceso de relleno de la misma. Apenas hay presencia de algunos de los residuos domesticos más habituales y previsibles. Además, la cantidad de fauna recuperada, una vez eliminados los restos pertenecientes a las carcasas de animales completos, podría considerarse exigua para un contexto excavado con meticulosidad si la formación de éste hubiese respondido a la eliminación de residuos procedentes de contextos residenciales a lo largo de un periodo prolongado. El hecho de que haya desaparecido toda evidencia sobre el entorno construido alrededor del pozo cuando todavía estaba en uso y tras su oclusión dificulta encontrar una respuesta apropiada a esos interrogantes. Es factible sospechar que la mayor parte de los elementos recuperados en el interior de la estructura hayan formado parte de la basura presente en sus inmediaciones (bajo un suelo entablado de madera, por ejemplo, o acumulado contra la parte superior externa de la estructura) y hayan acabado sólo por azar en su interior, al derrumbarse su cubierta y demolerse la coronación de sus muros perimetrales para terminar de ocluir sus restos.

\subsection{Datos relevantes y ruido de fondo}

Puede resultar desconcertante que la fecha tan baja obtenida para una de las fuentes de madera del estrato basal UE 26 (siglos VIII-X) no haya suscitado más debate. De acuerdo a las pautas metodológicas básicas, el contexto debería fecharse a partir del material más moderno contenido en él. Si por algún motivo ese dato nos alejara del resultado al que apunta el resto de las evidencias, lo razonable sería explorar las opciones que pudiesen ayudarnos a explicar o gestionar la incoherencia. En el marco de la alternativa propuesta a la lectura de la formación del contexto, nada conduce a pensar que se trate de una anomalía. Al contrario, si esas son las fechas más próximas al momento en que se amortizó el pozo, el verdadero trabajo pendiente consistiría en argumentar las razones por las que tantos otros materiales ofrecen fechas más altas.

Parece razonable sospechar que una parte del material antiguo, especiamente el de pequeñas dimensiones, pasara desapercibido durante el transcurso de las periódicas limpiezas que exigiría el mantenimiento regular de un pozo de agua para el consumo humano. A esa categoría de 'ruido de fondo' pertenecerían probablemente la mayor parte de las cerámicas, el material orgánico de pequeño tamaño y muchos de los restos óseos vinculados a formas de consumo. Sería aventurado en cambio procurar una explicación al hallazgo de restos de calzado, fenómeno registrado en otros pozos documentados en Europa occidental. Algunos han sugerido que podrían testimoniar ofrendas fundacionales, no siendo extraño que ofrezcan fechas considerablemente anteriores a la amortización de la estructura o que sean coetáneas a su construcción (Van Driel-Murray 1999: 137). No cabe decir lo mismo de las piezas de la vajilla de madera completas, de los cubos, la silla o de las carcasas de animales.

\section{CONCLUSIONES}

El presente trabajo parte de la premisa de que, dada su naturaleza fragmentaria, el documento arqueológico raras veces tiene una lectura única. Una modificación de la perspectiva, como la aplicada en este caso, implica cambios en el significado atribuible a un contexto. Por ello, abrir nuevas posibilidades de lectura e interpretación de los datos implica también alcanzar un conocimiento más maduro y crítico de la evidencia disponible. Frente a una inutilización natural, progresiva o casual de la estructura del pozo-depósito, algo que no exigiría (en principio) demasiadas explicaciones más allá de las sugeridas por los geólogos, se ofrece en estas páginas una lectura distinta que atribuye plena intencionalidad a la amortización repentina de un dispositivo funcionalmente crítico para un sitio como era la fortaleza del tómbolo de Cimadevilla.

La relectura de los datos que se ha propuesto tiene variadas implicaciones. A continuación se sugerirán algunas de las que pueden incidir más directamente en la historia de la villa gijonesa y del reino de Asturias durante el convulso periodo al que remite este espectacular contexto arqueológico.

\subsection{Gijón en la segunda mitad del primer milenio}

Casi un siglo y medio de opacidad informativa se extiende desde la noticia de la salida de la guarnición musulmana de Gijón (ca. 722 d.C.) a la del arribo de la primera flota de normandos (ca. 844 d.C.). Entre ambas, un llamativo silencio se cierne sobre el papel jugado por la principal fortaleza de la región (que la redacción Ovetense de la Crónica de Alfonso III denomina civitas) durante la constitución del reino de Asturias. Algunos han planteado que la historia de Gijón en los documentos escritos no comienza realmente hasta finales del siglo IX (García, Ríos 2013: 518). Los capítulos anteriores a esa fecha han sido trabajosamente construidos por la arqueología a partir de los fragmentos materiales del pasado, y es indudable que la labor arroja un saldo positivo. Es una satisfacción muy propia de arqueólogos comprobar cuánto pueden cambiar las cosas con cada intervención y con cada nuevo hallazgo, cómo se debe y puede reescribir la narración construida a partir de la evidencia arqueológica con cada pieza que se suma a las anteriores. A nadie se le escapa que el discurso arqueológico suele construirse sobre una irremisible condición de provisionalidad.

En una polémica aportación al debate sobre la historia de la villa, sus autores señalaban que a pesar de la machacona insistencia de algunos, "en Gijón no aparece ninguno de los elementos que definen un nú- 
cleo urbano romano" (García, Ríos 2013: 527). Antes de conocerse el hallazgo del pozo-depósito de Tabacalera pero habiendo trascendido noticias sobre la ocupación postimperial en la zona del Campo Valdés (Fernández Ochoa, Gil 2007; Fernández Ochoa y Morillo 2013), añadieron que "la vida de Cimadevilla se extingue en el siglo V", y que "la estratigrafía de Cimadevilla permite apreciar este hiato medieval, sobre el que se depositan los niveles y estructuras correspondientes a la fundación de la puebla en el último tercio del XIII" (Id.: 519). Los datos aportados por el pozo-depósito permiten constatar que, de haber existido un hiato ocupacional, su amplitud debería reducirse considerablemente. Las huellas de actividad no se extinguen en Cimadevilla al menos hasta bien avanzado el siglo VIII d.C.

En lo que respecta a la entidad del asentamiento, es preciso admitir que el circuito amurallado no otorga por sí solo una garantía sobre su carácter urbano. Aunque el lienzo torreado gijonés se asemeja bastante más al de algunas ciudades que al de otras fortificaciones de menor categoría, también es cierto que el imponente aspecto del acceso principal a la fortaleza, monumentalizado con torres en sillería, comparece también en enclaves, como el salmantino de Las Merchanas ( $\mathrm{J}$ ménez y Salinas 2013: 128-9), a los que no resultaría sencillo otorgar esa entidad.

Tampoco es fácil llegar a conclusiones firmes a partir de la labilidad del registro arqueológico gijonés tal y como ha llegado hasta nosotros. Su carácter esquivo lo comparte con la mayor parte de los registros de cronología altomedieval en casi cualquier establecimiento, sea o no urbano. En este caso particular, tal vez pueda justificarse teniendo en cuenta las probables consecuencias que acarreó el desmantelamiento de la muralla a finales del siglo XIV. Una vez desaparecido el principal elemento de contención de la estratigrafía intramuros, un cinturón contra el que es previsible que descansara el grueso de la ocupación doméstica del tómbolo, no sería de extrañar que sus restos quedaran expuestos a una intensa erosión, por la lluvia y las marejadas. Una parte de la estratificación arqueológica original pudo deslizar ladera abajo hasta dispersarse en el arenal y las marismas.

Es probable que la fortaleza de Gijón haya ejercido a lo largo de los primeros siglos altomedievales un papel relevante como centro de poder local, siendo un lugar de encuentro, celebración y mercado para la gente de la comarca y albergando la sede de un palatium y de una guarnición desde donde se ejercería el control político y fiscal del territorio. Serviría de puerto de escala en las rutas marítimas, modesto de tamaño, aunque tal vez de importancia relativa (suministros, aguada y refugio para embarcaciones). Podríamos concebir su entidad incluso como la de una pequeña ciuitas sine urbe (sensu Oller 2014), no muy distinta de la que pudo existir en Lugo de Llanera (Lucus Asturum) (Requejo 2014). La propuesta de García de Castro y Ríos, en el sentido de que en Gijón residiese solamente personal administrativo y promotores de actividades pesqueras y comerciales, podría no ir del todo desencaminada, sin embargo (García, Ríos 2013: 528). Las constricciones objetivas en lo que atañe al abastecimiento de agua dulce pudieron jugar un papel difícil de soslayar para que en el enclave se asentara de forma estable una población de tamaño significativo. La importancia de su estatus administrativo o del término que se elija para definirlo (castellum, civitas) tal vez deba pasar así a un segundo plano (Díaz, Menéndez 2016: 175).

\subsection{Posibles protagonistas y trascendencia histórica}

El registro material del contexto de amortización del pozo-depósito señala el carácter inequívocamente elitista de algunos de los residentes en el enclave gijonés. Es más, su carácter solamente podría corresponder a quienes ostentasen la más alta posición social y política en ese lugar y en ese tiempo: los gobernantes de la fortaleza. No es posible terciar desde la arqueología sobre si ese papel lo desempeñaban en su propio nombre o en el de una autoridad politica superior, cualquiera que fuese.

La tentación de vincular la fecha del evento que supuso la amortización del pozo con su contexto histórico y con los acontecimientos que nos han transmitido las fuentes escritas es ineludible. En la lacónica redacción de las crónicas, la selección tiende a amplificar lo trascendente y a minimizar lo trivial, pero también lo menos conveniente en términos políticos o ideológicos. Interpretar correctamente los silencios u omisiones puede ser a veces tan importante como analizar y comprender la selección o el propio contenido de las noticias.

La expulsión de la supuesta guarnición musulmana es la única noticia del siglo VIII referida a Gijón en las primeras crónicas asturianas. El acontecimiento pudo tener lugar entre 718 y 722 d.C. o ser incluso algo posterior $^{14}$. Tuvo que haber alguna buena razon por la que tras esa retirada y el acceso al poder de Pelayo la sede regia se estableciera en Cangas, y no en Gijón. Del mismo modo, cuando tras el asesinato de Fruela (768) el solio real sale de allí, los monarcas se asientan en Pravia (tal vez antes en Langreo) y finalmente en Oviedo, evitando recaer en el tómbolo de Cimadevilla ¿Qué ocurrió con el enclave gijonés para desaparecer del relato de las distintas crónicas entre (digamos) el año 720 y la arribada de normandos del 844? ¿Se trata de un silencio intencionado, como ocurre en otras ocasiones?

\footnotetext{
${ }_{14}$ De acuerdo a la interpretación de un pasaje de la Crónica Mozárabe, aquella cuya redacción es más proxima a los acontecimientos narrados, la fecha podría ser 737. El tope estaría en la sublevación bereber de 741-743 d.C. (Collins 1991: 136).
} 
De no haber permanecido más tiempo del oficialmente reconocido en manos enemigas, como se ha llegado a sugerir, ¿quizás lo estuvo en las de una facción opuesta al grupo dominante, el linaje que a la postre se impuso y fue responsable de la parte de la historia que nos ha sido transmitida? Tampoco se puede descartar que a partir de un cierto momento y por motivos que ignoramos, Gijón no fuera un lugar seguro ${ }^{15}$, o que no reuniera las condiciones mínimas de habitabilidad.

En principio, la lista de sospechosos sobre los que podría recaer la responsabilidad de una acción como la que provocó la inutilización del pozo y la hipotética destrucción de la sede político-militar en la que estaba integrado para unas fechas como las contempladas podría ceñirse a cuatro: piratas marítimos; una expedición de castigo de los ejércitos islámicos cordobeses; la población de esclavos o siervos que sabemos fue responsable de una revuelta digna de mención en las crónicas durante el reinado de Aurelio; o los propios astures o una facción de los mismos.

Las opciones de que la fortaleza gijonesa fuera objeto de algún ataque externo, bien por los musulmanes o por gentes del norte (anticipándose a las primeras referencias documentales a los raids normandos ${ }^{16}$ ) no se pueden descartar, aunque tampoco tienen un soporte real. Dentro del rango temporal contemplado, la década de los cuarenta del siglo octavo conoció la sublevación de los beréberes frente a los gobernadores nombrados por Damasco. Poco después, en el año 756, alcanzaría el poder en Córdoba Abderramán I, primer emir de la dinastía omeya andalusí. Cuentan las crónicas que ocupó los treinta años de su gobierno consolidando su poder frente a los partidarios del anterior emir y sus hijos, y de las distintas facciones políticas y tribales, sin prestar excesiva atención a la situación política fuera de sus fronteras. Si nos moviésemos en torno al reinado de Fruela I (757-768), las fuentes se refieren a tensiones políticas y rebeliones en Galicia y Vasconia. No parece de recibo que, tras el largo reinado de Alfonso I, Gijón quedara tan lejos todavía para la dinastía canguesa como para no formar parte del núcleo del reino. Se desconoce igualmente si pudieron llegar a Gijón las expediciones de castigo lanzadas desde Córdoba en 766-767 o 791.

Una tercera posibilidad, por remota que parezca, vincularía la amortización del pozo a la revuelta de esclavos durante el reinado de Aurelio (768-774), en el caso de que ésta hubiera tenido en Gijón uno de sus escenarios. Las razones por las que un episodio de esa naturaleza trascendió a unas crónicas habitualmente tan parcas en detalles han sido motivo de especulaciones
(Isla 2015: 253, n.8), y en otras sólo se ha citado el episodio para lamentar el laconismo de la fuente (Wickham 2007: 47; 2005: 584). Diversos apriorismos han condicionado la lectura de la noticia (BESGA 2000: 362-79).

Llegamos así a la cuarta de las hipótesis. La posibilidad de que una facción de los propios astures pudiera haber decidido inutilizar la fortaleza de Gijón a fin de evitar la radicación en ella de un poder hostil (o de que se entablasen desde allí pactos con el enemigo) sería una alternativa adicional a las antes expuestas. Una circunstancia así sería compatible con la parquedad informativa que mantienen las crónicas sobre Gijón, sin duda uno de los polos políticos más activos del Cantábrico durante el siglo VII y el más significativo de Asturias antes de la fundación de Oviedo ${ }^{17}$. Tras abandonar Cangas, es posible que Aurelio trasladara su corte al concejo de Langreo, y se sabe que Silo escogió luego Pravia. El denostado Mauregato (783-788), bastardo de Alfonso I, parece el único personaje ajeno al linaje reinante, tal vez relacionado con quienes asesinaron en Cangas a Fruela I. Para sustituirle en el poder, los magnates escogieron a un sobrino de Alfonso I (Vermudo), que al cabo de tres años abdicó en favor de Alfonso II, refugiado en tierras de su familia alavesa. Cualquier hipotética vinculación de Mauregato y su madre con la familia gobernante en Gijón debe considerarse especulativa. Resulta imposible de contrastar si los integrantes de ese linaje pudieron ser los causantes de los problemas durante la minoría de edad de Alfonso II. Lo único cierto es que, al cabo de ese periodo de turbulencias políticas, el nuevo rey Alfonso II (791-842) fijó su sede en Oviedo, de donde ya no se movería hasta ser trasladada la corte a León (Figura 6).

En todo caso, la relativa confianza que merezca el escenario en que pudiera haberse producido la inutilización del pozo debe pasar a un segundo plano frente a la tozuda materialidad de los datos arqueológicos. Estos señalan la presencia de artefactos y ecofactos fechables al menos hasta mediados del siglo VIII d.C. en el depósito basal. El supuesto podría ser falsado mediante la datación radiocarbónica de los restos óseos de algunos de los animales arrojados al pozo, o de los que poco más tarde cayeron en él como en una trampa, como pudiera ser el caso de algunas de las aves. La propuesta, por tanto, sería susceptible de contrastación empírica. Las alternativas expuestas (u otras posibles) han de valorarse en función de su grado de verosimilitud teniendo en cuenta las circunstancias históricas específicas en el espacio y en el tiempo en las que cabría situar el hallazgo y la información disponible procedente de las fuentes narrativas.

\footnotetext{
15 Todas estas cuestiones han sido planteadas desde antiguo, véase p.ej. GONZÁLEZ 1979: 99, n. 25. Sobre los silencios de las crónicas: Gil et alii 1985: 71-ss.

${ }^{16}$ Sánchez Pardo (2010: 60-1) alude a las discutidas teorías de Arne Melvinger sobre expediciones en el siglo VIII. Véase también Scheen, 1996. ${ }^{17}$ La supuesta colaboración del linaje gobernante en Gijón con el enemigo cordobés se señala como posible causa del postergamiento político de la villa en Menéndez Bueyes 2001: 299. Es intrigante también la omisión del sitio cuando durante el reinado de Alfonso III se señala la fortificación de núcleos costeros como Gauzón y Torres del Oeste, o el propio castillo de Oviedo.
} 


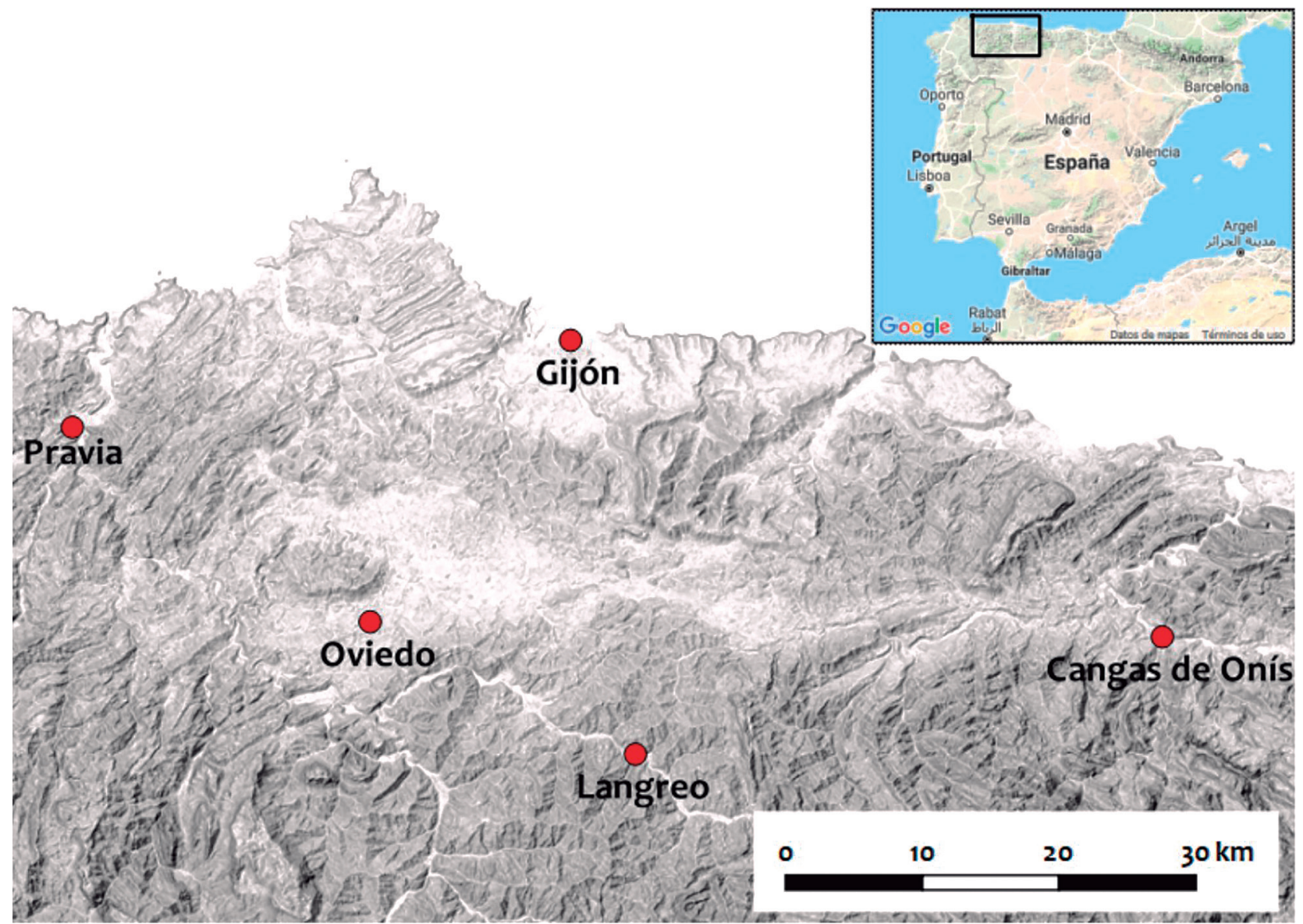

Fig. 6. Gijón y las sedes de la monarquía asturiana en el siglo VIII d.C. / Gijón and the royal sees of the Asturian monarchy in the eighth century AD.

\subsection{Perspectivas}

Como se ha argumentado en las páginas previas, los datos publicados del contexto del pozo-depósito de Tabacalera son lo suficientemente prolijos como para armar una lectura alternativa tanto del proceso formativo del depósito arqueológico como de su cronología. Además del proceso plurisecular defendido hasta ahora, es posible sostener que un evento de naturaleza violenta haya podido ser el detonante de la inutilización y la posterior obliteración del pozo. En lo que respecta al momento en que sucedió, se ha propuesto una fecha inmediatamente posterior al ecuador del siglo VIII como aquella en la que mejor encajan los diversos elementos del rompecabezas formado por los materiales contenidos en los estratos basales del depósito y las determinaciones radiocarbónicas disponibles.

Una vez reunidos los distintos ingredientes y leídos de forma unitaria pueden cobrar un nuevo significado. El trono, el laúd, la mascota egipcia, la jauría de perros... aportan un testimonio difícilmente discutible de lo que arqueológicamente puede entenderse como un contexto social elitista, en este caso de un destacable nivel. Se adhieren, en suma, a las definidas como formas de representación del poder, elementos materiales que visibilizan la distinción, el prestigio o el estatus exclusivo de ciertos individuos. El que todas esas piezas hayan aparecido juntas en el fondo del pozo sugiere que éste no fue probablemente una simple estructura de uso doméstico, sino una infraestructura esencial para la habitabilidad del enclave, ubicada probablemente en el patio de la residencia de quien gobernaba la fortaleza en aquellos días, en el centro de lo que podría haber sido su palacio. Mientras que el grueso de ese conjunto de hallazgos remite a esa residencia principal del enclave amurallado gijonés del siglo octavo, es indudable que otros muchos forman parte del ruido de fondo generado por una ocupación plurisecular previa del área.

La caracterización del contexto en los términos sociales y políticos que se han señalado nos coloca frente a una serie de nuevos dilemas. El primero atañe a la invisibilidad de Gijón en las primeras crónicas asturianas. No es posible encontrar en ellas pistas acerca de lo sucedido, y resulta intrigante que el núcleo gijonés no haya jugado el papel que tal vez cabría esperar en la historia del periodo formativo del reino de Asturias. El segundo concierne a la identificación de los responsables de una acción destructiva de esa naturaleza. En un apartado anterior se han barajado diversas alternativas, algunas externas y otras de orden interno, pero tampoco es descartable un abandono de la fortaleza forzado 
por las circunstancias. Ante la dificultad o imposibilidad de su defensa, podría haber sido desalojada por sus ocupantes, dejando inutilizado el suministro de agua potable para que cualquier fuerza hostil viera entorpecido su eventual asiento.

El presente trabajo entiende que es posible maximizar el potencial heurístico del análisis arqueológico aplicable a este caso y explorar sus alternativas. Es seguro que no se colman en estas páginas todas las posibilidades que se abren al analizar el contexto arqueológico descubierto en Cimadevilla desde nuevos enfoques. Que la propuesta sirva de revulsivo para continuar extrayendo de los hallazgos la riqueza informativa que todavía guardan ha sido un objetivo explícito.

\section{AGRADECIMIENTOS}

Trabajo realizado en el marco del Proyecto "Agencia campesina y complejidad sociopolítica en el noroeste de la Península Ibérica en época medieval" (Ministerio de Economía, Industria y Competitividad, AEI/ FEDER UE HUM2016-76094-C4-2-R) y del Grupo de investigación reconocido (GIR) ATAEMHIS, de la Universidad de Salamanca.

Agradezco a los revisores anónimos sus valiosas sugerencias, de las que se ha beneficiado el texto.

\section{BIBLIOGRAFÍA}

Alba Calzado, M., Gutiérrez Lloret, S., 2008. Las producciones de transición al mundo islámico: el problema de la cerámica paleoandalusí (siglos VIII y IX). En: Bernal D., Ribera, A. (Eds.), Cerámicas hispanorromanas. Un estado de la cuestión, 585613. Universidad de Cádiz, Cádiz.

Alonso Ojeda, R., 2015. El acuífero del Cerro de Santa Catalina. En: Fernández Ochoa, C., Orejas, A., García, P., Gil, F. (Eds.), La Fábrica de Tabacos de Gijón. Arqueología e historia de un espacio milenario, 343-347. Ayuntamiento de Gijón, Gijón.

Amorós Ruiz, V., 2011. Contextos cerámicos del siglo VIII en el Tolmo de Minateda.. Instituto de Estudios Albacetenses 'Don Juan Manuel', Albacete. Serie I, Estudios 198.

Besga Marroquín, A., 2000. Orígenes hispano-godos del reino de Asturias. RIDEA, Oviedo. Fuentes y estudios de historia de Asturias 21

Blanco González, H., 2003. La ciudad del agua. Historia del abastecimiento público de agua en Gijón. EMA-Ayuntamiento, Gijón.

Bonifay, M., 2004. Etudes sur la céramique romaine tardive d'Afrique. Archaeopress, Oxford. BAR International Series 1301.

Collins, R., 1991. La conquista árabe, 710-797. Crítica, Barcelona (ed. orig. The Arab conquest of Spain 710-797, Basil Blackwell, Oxford, 1989).

Díaz Martínez, P.C., Menéndez Bueyes, L.R., 2016. Romanos, visigodos e indígenas : las comunidades del norte de Hispania en los inicios de la Edad Media (cuarenta años después). Anejos de Nailos 3, 161-189.
Fernández Fernández, A., 2014. El comercio tardoantiguo (ss. IV-VII) en el Noroeste peninsular a través del registro cerámico de la Ría de Vigo. Archaeopress, Oxford.

Fernández Ochoa, C., 1994. Excavaciones arqueológicas en Gijón. Una industria de salazones de época romana en la Plaza del Marqués. Ayuntamiento de Gijón, Gijón.

Fernández Ochoa, C., 1997. La muralla romana de Gijón (Asturias). Electa, Gijón.

Fernández Ochoa, C., Gil Sendino, F., 2007. El recinto amurallado de Gijón. Origen y permanencia hasta la Edad Media". En: Rodríguez Colmenero, A., Rodá de Llanza, I. (Eds.), Murallas de ciudades romanas en el occidente del Imperio. Lucus Augusti como paradigma, 403-414. Diputación provincial de Lugo, Lugo.

Fernández Ochoa, C., Morillo Cerdán, A., 2013. Oceanus Hispanus: navegación y comercio a orillas del Atlántico en época romana. En: Morais, R., Granja, H., Morillo, A. (Eds.), O irado mar Atlántico: o naufragio bético augustano de Esposende (Norte de Portugal), 57-97. Museu Diego de Sousa, Braga.

Fernández Ochoa, C., Orejas Saco Del Valle, A., García Díaz, P., Gil Sendino, F. (Eds.), 2015a. La Fábrica de tabacos de Gijón. Arqueología e historia de un espacio milenario. Ayuntamiento de Gijón, Gijón.

Fernández Ochoa, C., García Díaz, P., Gil Sendino, F., Orejas Saco del Valle, A., 2015b. El registro arqueológico. Excavaciones en el sector 20. En: FERNÁNDEZ OCHOA, C., OREJAS, A., GARCÍA, P., GIL, F. (Eds.), La Fábrica de Tabacos de Gijón. Arqueología e historia de un espacio milenario, 114-125. Ayuntamiento de Gijón, Gijón.

Fernández Ochoa, C., Salido Domínguez, J., García Díaz, P., Gil Sendino, F., 2015c. Estudio de los materiales arqueológicos. En: Fernández Ochoa, C., Orejas, A., García, P., Gil, F. (Eds.), La Fábrica de Tabacos de Gijón. Arqueología e historia de un espacio milenario, 126-157. Ayuntamiento de Gijón, Gijón.

Fernández Ochoa, C., Orejas Saco del Valle, A., Gil Sendino, F., 2015d. Arquitectura y función del pozo-depósito de Tabacalera. En: Fernández Ochoa, C., Orejas, A., García, P., Gil, F. (Eds.), La Fábrica de Tabacos de Gijón. Arqueología e historia de un espacio milenario, 158-181. Ayuntamiento de Gijón, Gijón.

Fernández Ochoa, C., Salido Domínguez, J., García Díaz, P., Gil Sendino, F., 2015e. El uso de la madera en el periodo tardoantiguo: objetos de la vida cotidiana procedentes de la excavación de la Fábrica de Tabacos de Gijón (Asturias). Zephyrus 76, 141-158.

Fischer, S., Lind, L., 2017. Late Roman Gaul: survival amidst collapse? In: Cunningham, T., Driessen, J. (Eds.), Crisis to collapse. The archaeology of social breakdown, 99-130. [Aegis 11]. Presses Universitaires, Louvain.

García de Castro, F.J., Ríos González, S., 2013. Consideraciones en torno a la historia de Gijón en la Edad Antigua. En: De La Rasilla, M. (Coord.). F. Javier Fortea Pérez. Universitatis Ovetensis Magister. Estudios en homenaje, 515-532. Ménsula Ediciones, Oviedo.

Gil Fernández, J., Moralejo, J.L., Ruiz de la Peña, J.I., 1985. Crónicas asturianas. Universidad de Oviedo, Oviedo.

González y Fernández-Vallés, J.M., 1979. Pravia, 'capital' del reino asturiano. Asturiensia medievalia 3, 87-104.

Isla Frez, A., 2015. The aristocracy and the monarchy in Northwestern Iberia between the eighth and the eleventh century. In: D'Emilio, J. (Ed.), Culture and society in medieval Galicia. A 
cultural crossroads at the edge of Europe, 251-280. Brill, Leiden-Boston.

Jiménez González, M., Salinas de Frías, M., 2013. Territorio vetón: aspectos históricos, patrimoniales y culturales". En: Salinas M. (Coord.), Interpretar la frontera. Jornadas de patrimonio, turismo y desarrollo local, 127-146. Diputación, Salamanca.

Jiménez Jáimez, V., 2008. El ciclo formativo del registro arqueológico: una alternativa a la dicotomía deposicional/postdeposicional. Zephyrus 62(2), 125-137.

Jiménez-Sánchez, M., Naves-Ramos, B., Ballesteros, D., Giralt, S., Moreno, A., Valero-Garcés, B., Jiménez Muñoz, V., 2015. El relleno sedimentario del pozo-depósito de la antigua Fábrica de Tabacos de Gijón: un archivo geológico de los siglos VI-VIII. En: Fernández Ochoa, C., Oejas, A., García, P., Gil, F. (Eds.), La Fábrica de Tabacos de Gijón. Arqueología e historia de un espacio milenario, 184-193. Ayuntamiento de Gijón, Gijón.

Kacki, S., Réveillas, H., Sachau-Carcel, G., Giuliani, R., Blanchard, Ph., Castex, D., 2014. Réévaluation des arguments de simultanéité des dépôts de cadavres: l'exemple des sépultures plurielles de la catacombe des Saints Pierre-et-Marcelin (Rome). Bulletins et Mémoires de la Société d'Anthropologie de Paris 26 (1), 88-97.

Lawrence, T.E., 1955. Los siete pilares de la sabiduría. Editorial Sur, Buenos Aires ( $2^{\mathrm{a}}$ ed.) (ed. orig. Seven pillars of wisdom, London, 1928).

Loveluck, C., 2009. The dynamics of elite lifestyles in the 'Rural World', AD 600-1150: archaeological perspectives from Northwest Europe. In: Bougard, F., Le Jan, R., Mckitterick, R. (Eds.), 139-170. La culture du Haut Moyen Âge. Une question d'élites? Brepols, Turnhout.

Loveluck, C., 2011. Problems of the definition and conceptualisation of Early Medieval elites, AD 450-900 : the dynamics of the archaeological evidence. In: Bougard, F., Werner H., Le Jan, R. (Eds.), Théorie et pratiques des élites au Moyen Âge, 21-68. Brepols, Turnhout.

Lucas, G., 2012. Understanding the archaeological record. Cambridge University Press, Cambridge.

Llorente Rodríguez, L., Sánchez Marco, A., Nores Quesada, C., Morales Muñiz, A., 2015a. La fauna del pozo-depósito romano de la Fábrica de Tabacos de Gijón. Aspectos generales de las unidades estratigráficas 25 y 26. En: Fernández Ochoa, C., Orejas, A., García, p., gil, F. (Eds.), La Fábrica de Tabacos de Gijón. Arqueología e historia de un espacio milenario. Ayuntamiento de Gijón, Gijón, 226-237.

Llorente Rodríguez, L., Nores Quesada, C., Gaubert, P., Morales Muñiz, A., 2015b. ¿Nativo o foráneo? El meloncillo de Tabacalera y su aportación a la historia de la especie. En: Fernández Ochoa, C., Orejas, A., García, P., Gil, F. (Eds.), La Fábrica de Tabacos de Gijón. Arqueología e historia de un espacio milenario, 246-251. Ayuntamiento de Gijón, Gijón.

Llorente Rodríguez, L., Morales Muñiz, A., 2015. Los moluscos del pozo-depósito de Tabacalera. En: Fernández Ochoa, C., Orejas, A., García, P., Gil, F. (Eds.), La Fábrica de Tabacos de Gijón. Arqueología e historia de un espacio milenario, 260-265. Ayuntamiento de Gijón, Gijón.

Menéndez Bueyes, L.R., 2001. Reflexiones críticas sobre el origen del reino de Asturias. Universidad de Salamanca, Salamanca.

Metcalfe, N., 2002. A short history of biological warfare. Medicine, conflict and survival, 18(3), 271-282. https://doi. org/10.1080/13623690208409635
Morales Muñiz, A., Casado Violat, S., López Arrabé, J., Llorente Rodríguez, L., 2015. Caracterización morfométrica de los perros de la unidad estratigráfica 26 del pozo-depósito de la Tabacalera (Canis familaris). En: Fernández Ochoa, C., Orejas, A., García, P., Gil, F. (Eds.), La Fábrica de Tabacos de Gijón. Arqueología e historia de un espacio milenario, 238-245. Ayuntamiento de Gijón, Gijón.

Oller Guzmán, J., 2014. La civitas sine urbe y su función de vertebración en el territorio provincial hispano: los casos de Egara y Caldes de Montbui. Pyrenae 45 (1), 89-110.

Price, N., 2012. Wooden worlds. Individual and collective in the chamber graves of Birka. In: Hedenstierna-Jonson, C. (Ed.), Birka nu. Pågående forskning om världsarvet Birka och Hovgården, 81-93. Historiska Museet, Stockholm. The National Historical Museum, Stockholm Studies 22.

Quirós castillo, J.A., 2013. "Archaeology of power and hierarchies in Early Medieval villages in Northern Spain". In: Klápste, J. (Ed.), Hierarchies in rural settlements, 199-212. Brepols, Turnhout. Ruralia IX.

Requejo Pagés, O., 2014. El enclave romano de Lucus Asturum. Perxuraos, revista cultural de Llanera 3, 7-15.

Requejo Pagés, O., 2016. Cerámica altomedieval de Asturias. En: Vigil-Escalera, A., Quirós, J.A. (Eds.), La cerámica de la Alta Edad Media en el cuadrante Noroeste de la península Ibérica (siglos V-X), 113-138. UPV/EHU, Bilbao.

Roselló Izquierdo, E., Morales Muñiz, A., 2015. Los peces del pozo-depósito de Tabacalera (Unidad estratigráfica 26). En: Fernández Ochoa, C., Orejas, A., García, P., Gil, F. (Eds.), La Fábrica de Tabacos de Gijón. Arqueología e historia de un espacio milenario, 252-259. Ayuntamiento de Gijón, Gijón.

Sánchez Díaz, J., Asins Velis, S., Marco Segura, J.B., 2015. Análisis sedimentológico del pozo depósito de agua de la Tabacalera de Gijón. Interpretación de los resultados. En: Fernández Ochoa, C., Orejas, A., García, P., Gil, F. (Eds.), La Fábrica de Tabacos de Gijón. Arqueología e historia de un espacio milenario, 194-199. Ayuntamiento de Gijón, Gijón.

Sánchez López, E.H., 2018. The role of fresh water in fish processing in Antiquity. Journal of Maritime archaeology (2018), doi: 10.1007/s11457-018-9193-3.

Sánchez-Pardo, J.C., 2010. Los ataques vikingos y su influencia en la Galicia de los siglos IX-XI". Anuario Brigantino 33, 57-86.

Scheen R., 1996. Viking raids on the spanish peninsula. Militaria, revista de cultura militar 8, 67-88.

Schiffer, M.B., 1986. Radiocarbon dating and the 'Old Wood' problem: the case of the Hohokam chronology. Journal of Archaeological Science 13, 13-30.

Schiffer, M.B., 1987. Formation processes of the archaeological record. University of Utah press, Salt Lake City.

Serrano Herrero, E., Torra Pérez, M., Catalán Ramos, R., Vigil-Scalera Guirado, A., 2016. La cerámica de los siglos VIII-IX en Madrid, Toledo y Guadalajara". En: Vigil-Escalera, A., Quirós, J.A. (Eds.), La cerámica de la Alta Edad Media en el cuadrante Noroeste de la península Ibérica (siglos V-X) , 279313. UPV/EHU, Bilbao.

Van Driel-Murray, C., 1999. And did those feet in ancient time... feet and shoes as a material projection of the self". In: Baker, P., Forcey, C., Jundi, S., Witcher, R. (Eds.), TRAC 98. Proceedings of the Eighth Annual Theoretical Roman Archaeology Conference, Leicester 1998, 131-140. Oxbow, Oxford. doi: http://doi.org/10.16995/TRAC1998_131_140 
Vigil-Escalera Guirado, A., Quirós Castillo, J.A., 2016. La cerámica de la Alta Edad Media en el Noroeste peninsular: una introducción. En: Vigil-Escalera, A, Quirós, J.A. (Eds.), La cerámica de la Alta Edad Media en el cuadrante Noroeste de la península Ibérica (siglos V-X), 23-41. UPV/EHU, Bilbao.

Walton, D.N., 2006. Poisoning the well. Argumentation 20, 273-307.

Wanscher, O., 1980. Sella Curulis. The folding stool an ancient symbol of dignity. Rosenkilde, Copenhage.

Wickham, C., 2007. Espacio y sociedad en los conflictos campesinos en la Alta Edad Media. En: Rodríguez López, A.M. (Ed.), El lugar del campesino. En torno a la obra de Reyna Pastor, 33-60. CSIC-Universidad de Valencia, Valencia.

Willemsen, A., 2015. Looking for early-medieval royal graves in the Low Countries and the Rhineland. In: Willemsen, A., Kik, H. (Eds.), Golden Middle Ages in Europe. New research into early-medieval communities and identities, 15-23. Brepols, Turnhout. 
